\title{
Duality for discrete integrable systems II
}

\author{
Peter H. van der Kamp ${ }^{1,3}$, G.R.W Quispel ${ }^{1}$, Da-jun Zhang ${ }^{2}$ \\ ${ }^{1}$ Department of Mathematics and Statistics \\ La Trobe University, Victoria 3086, Australia \\ ${ }^{2}$ Department of Mathematics, Shanghai University \\ Shanghai 200444, China \\ ${ }^{3}$ Corresponding author, P.vanderKamp@LaTrobe.edu.au
}

\begin{abstract}
We generalise the concept of duality to lattice equations. We derive a novel 3 dimensional lattice equation, which is dual to the lattice AKP equation. Reductions of this equation include Rutishauser's quotient-difference (QD) algorithm, the higher analogue of the discrete time Toda (HADT) equation and its corresponding quotientquotient-difference (QQD) system, the discrete hungry Lotka-Volterra system, discrete hungry QD, as well as the hungry forms of HADT and QQD. We provide three conservation laws, we conjecture the equation admits $\mathrm{N}$-soliton solutions and that reductions have the Laurent property and vanishing algebraic entropy.
\end{abstract}

Keywords: 3D lattice equation, integrable, duality, AKP, conservation laws, solitons, quotients-difference system.

\section{Introduction}

Our aim in the present paper is threefold:

1. To generalise the concept of duality (introduced in [29] for ordinary difference equations) to lattice equations;

2. To use duality to derive the 3 dimensional (3D) lattice equation

$$
\begin{aligned}
0= & a_{1}\left(\tau_{k-1, l+1, m+1} \tau_{k+1, l, m} \tau_{k+1, l, m+1} \tau_{k+1, l+1, m}-\tau_{k, l, m+1} \tau_{k, l+1, m} \tau_{k, l+1, m+1} \tau_{k+2, l, m}\right) \\
& +a_{2}\left(\tau_{k, l+1, m} \tau_{k, l+1, m+1} \tau_{k+1, l-1, m+1} \tau_{k+1, l+1, m}-\tau_{k, l, m+1} \tau_{k, l+2, m} \tau_{k+1, l, m} \tau_{k+1, l, m+1}\right) \\
& +a_{3}\left(\tau_{k, l, m+1} \tau_{k, l+1, m+1} \tau_{k+1, l, m+1} \tau_{k+1, l+1, m-1}-\tau_{k, l, m+2} \tau_{k, l+1, m} \tau_{k+1, l, m} \tau_{k+1, l+1, m}\right) \\
& +a_{4}\left(\tau_{k, l, m} \tau_{k, l+1, m+1} \tau_{k+1, l, m+1} \tau_{k+1, l+1, m}-\tau_{k, l, m+1} \tau_{k, l+1, m} \tau_{k+1, l, m} \tau_{k+1, l+1, m+1}\right) .
\end{aligned}
$$

3. To provide conservation laws for equation (1), to present reductions to two dimensional integrable systems, and to support our conjecture that equation (1) admits $N$-soliton solutions and its reductions have the Laurent property and vanishing algebraic entropy.

Most of currently known integrable 3D lattice equations are related to discretizations of the three continuous 3D Kadomtsev-Petviashvili equations called AKP, BKP and CKP. The lattice AKP equation,

$$
A \tau_{k+1, l, m} \tau_{k, l+1, m+1}+B \tau_{k, l+1, m} \tau_{k+1, l, m+1}+C \tau_{k, l, m+1} \tau_{k+1, l+1, m}=0,
$$

was first derived by Hirota [18, and is also called the Hirota-Miwa equation [28. The more general lattice BKP equation (also called the Miwa equation),

$$
A \tau_{k+1, l, m} \tau_{k, l+1, m+1}+B \tau_{k, l+1, m} \tau_{k+1, l, m+1}+C \tau_{k, l, m+1} \tau_{k+1, l+1, m}+D \tau_{k, l, m} \tau_{k+1, l+1, m+1}=0
$$

was first found by Miwa in [28]). The lattice CKP equation,

$$
\begin{aligned}
& \left(\tau_{k, l, m} \tau_{k+1, l+1, m+1}+\tau_{k+1, l, m} \tau_{k, l+1, m+1}-\tau_{k, l+1, m} \tau_{k+1, l, m+1}-\tau_{k, l, m+1} \tau_{k+1, l+1, m}\right)^{2} \\
= & 4\left(\tau_{k, l, m} \tau_{k+1, l, m+1}-\tau_{k, l+1, m} \tau_{k, l, m+1}\right)\left(\tau_{k+1, l, m} \tau_{k+1, l+1, m+1}-\tau_{k+1, l+1, m} \tau_{k+1, l, m+1}\right)
\end{aligned}
$$


was first derived by Kashaev as a 3D lattice model associated with the local Yang-Baxter relation 22, and later was independently found by King and Schief [23] as a superposition principle for the continuous CKP equation. This equation is also formulated as a hyperdeterminant in 33 .

The AKP equation is a bilinear equation on a six-point octahedral stencil ( $A_{3}$ lattice). Equations of this type have been classified with respect to multi-dimensional consistency in 1]. The lattice BKP and CKP equations are both defined on an 8-point cubic stencil. However, whereas lattice BKP is bilinear, the lattice CKP is quartic and nonlinear. A nonlinear form of the AKP equation (quartic and defined on a 10-point stencil) was given in [13, equation 5.5]. A quintic nonlinear non-potential lattice AKP equation was given in [11, equation 3.19]. This equation is defined on a 10-point stencil [11, Figure 3]. A quadrilinear 3D lattice equation related to the lattice BKP equation, defined on a 14-point stencil ( $D_{3}$ lattice), is presented in [23, Equation 24]. Our equation (11), which we will obtain as a dual to the AKP equation (2), is a quadrilinear equation defined on the 14 point stencil depicted in Figure 1. To our knowledge equation (1) is new. Given that the number of known integrable 3D lattice equations

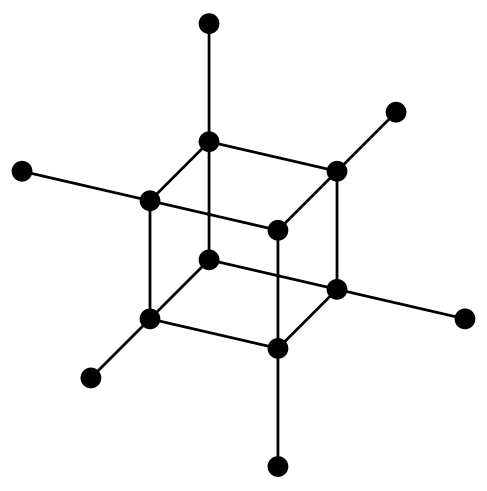

Figure 1: The 14-point stencil of equation (1).

is quite small, cf. [16, Sections 3.9-3.10], any possible addition to this number would seem worthwhile pursuing.

The idea of duality for ordinary difference equations is as follows: given an ordinary difference equation $(\mathrm{O} \Delta \mathrm{E})$, $E=E\left(u_{n}, u_{n+1}, \ldots, u_{n+d}\right)=0$, with an integral, $K[n]=K\left(u_{n}, u_{n+1}, \ldots, u_{n+d-1}\right)$, the difference of the integral with its upshifted version factorises $K[n+1]-K[n]=E \Lambda$. The quantity $\Lambda$ is called an integrating factor. The equation $\Lambda=0$ is a dual equation to the equation $E=0$, both equations share the same integral. If $E=0$ has several integrals $K_{i}$, then a linear combination of them gives rise to a dual with parameters:

$$
\sum_{i} a_{i} K_{i}[n+1]-\sum_{i} a_{i} K_{i}[n]=E\left(\sum_{i} a_{i} \Lambda_{i}\right) .
$$

In 29] duals to $(d-1,-1)$-periodic reductions of the modified Korteweg-de Vries (mKdV) lattice equation are shown to be integrable maps, namely level-set-dependent mKdV maps. In 4 a novel hierarchy of maps is found by applying the concept of duality to the linear equation $u_{n}=u_{n+d}$, and $\left\lfloor\frac{d-1}{2}\right\rfloor$ integrals are provided explicitly. The integrability of these maps is established in [20. We note that dual equations are not necessarily integrable, examples exist where the dual is not integrable [5]. In [21, the authors study several integrable 4th order maps and integrable maps that are dual to them.

Given a 2D lattice equation, $E=E\left(u_{k, l}, \ldots, u_{k+d, l+e}\right)=0$, instead of considering differences of integrals we now consider conservation laws:

$$
P[k+1, l]-P[k, l]+Q[k, l+1]-Q[k, l]=E \Lambda .
$$

Here the quantity $\Lambda$ is called the characteristic of the conservation law. Again the equation $\Lambda=0$ or a linear combination, $\sum_{i} a_{i} \Lambda_{i}=0$, can be viewed as the dual equation to $E=0$. The situation for 3D lattice equations is similar.

The structure of the paper is as follows. In section 2 we present a $3 \mathrm{D}$ lattice equation which is dual to the lattice AKP equation, and we provide a matrix formula which simultaneously captures four conservation laws for the AKP equation as well as three conservation laws for the dual AKP equation. In section 3 we show that these conservation laws give rise to quotients-difference formulations, in the same way that Rutishauser's quotientdifference (QD) algorithm [30] is a quotient-difference formulation of the discrete-time Toda equation [19]. In 
section 4 we provide 1-soliton and 2-solition solutions, and we provide evidence to support a conjectured form for the general $N$-soliton solution. In section 5 we provide evidence to support our conjecture that 2-periodic reductions to ordinary difference equations have the Laurent property. In section 6 we provide details of calculations which indicate that 2-periodic reductions of the dual AKP equation have quadratic growth. In section 7 we show that reductions of the dual AKP equation (1) to 2D lattice equations include the higher analogue of the discrete time Toda (HADT) equation and its corresponding quotient-quotient-difference (QQD) system [32], the discrete hungry Lotka-Volterra system, discrete hungry QD, as well as the hungry forms of HADT and QQD introduced in [3].

\section{Derivation of a dual to the lattice AKP equation, and a matrix conservation law}

Seven characteristics of conservation laws for the lattice AKP equation can be obtained from the results in [26]. We choose to only consider the parameter independent ones and we set all arbitrary functions equal to 1 . We will denote shifts in $k$ using tildes, shifts in $l$ by hats, and shifts in $m$ by dots, e.g. $\hat{\tilde{\tau}}=\tau_{k+1, l+1, m-1}$. The four characteristics (denoted $\Lambda_{1}, \Lambda_{2}, \Lambda_{3}, \Lambda_{7}$ in [26]) are given by

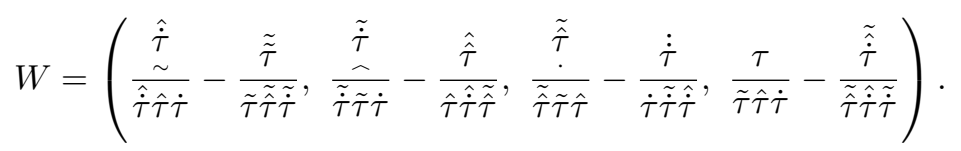

One can now check the following matrix conservation law

$$
\tilde{P}-P+\hat{Q}-Q+\dot{R}-R=V^{T} W
$$

where $P, Q, R$ are the $3 \times 4$ matrices

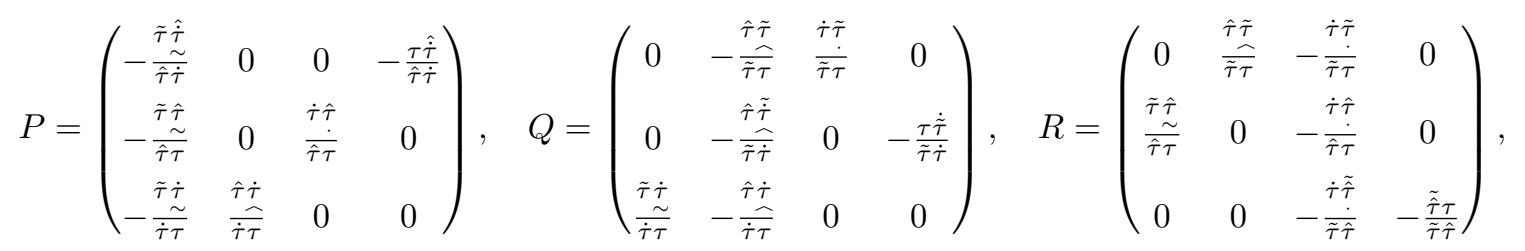

and $V^{T}$ denotes the transpose of

$$
V=(\hat{\dot{\tau}} \tilde{\tau}, \tilde{\dot{\tau}} \hat{\tau}, \hat{\tilde{\tau}} \dot{\tau}) .
$$

Denoting two vectors of coefficients by $X=(A, B, C)$ and $Y=\left(a_{1}, a_{2}, a_{3}, a_{4}\right)$, we have that $X V^{T}=0$ represents the AKP equation 2 and the equation $W Y^{T}=0$ is equivalent to equation (1).

Hence, pre-multiplying (5) with $X$ gives four conservation laws for the lattice AKP equation, and postmultiplying (5) with $Y^{T}$ yields three conservation laws for equation (1). Thus the lattice AKP equation and equation (1) are dual to each other.

\section{Corresponding quotients-difference systems}

The lattice AKP equation and the dual AKP equation can each be written as a system of one difference equation combined with a number of quotient equations.

Let us introduce variables

$$
p=\frac{\tau \hat{\dot{\tau}}}{\hat{\tau} \dot{\tau}}, \quad q=\frac{\tau \dot{\tilde{\tau}}}{\tilde{\tau} \dot{\tau}}, \quad v=\frac{\tilde{\hat{\tau}} \tau}{\tilde{\tau} \hat{\tau}} .
$$

Note that $p=-P_{14}, q=-Q_{24}$ and $v=-R_{34}$. Hence, the fourth conservation law for the AKP equation can be written as the difference equation

$$
A(\tilde{p}-p)+B(\hat{q}-q)+C(\dot{v}-v)=0 .
$$

Taking logarithms, we find

$$
\begin{aligned}
& \ln (p)=c+\hat{\dot{c}}-\hat{c}-\dot{c}=(1-\hat{S})(1-\dot{S}) c \\
& \ln (q)=c+\tilde{\tilde{c}}-\tilde{c}-\dot{c}=(1-\tilde{S})(1-\dot{S}) c \\
& \ln (v)=c+\tilde{\tilde{c}}-\tilde{c}-\hat{c}=(1-\tilde{S})(1-\hat{S}) c
\end{aligned}
$$


where $c=\ln (\tau)$, capital $\tilde{S}$ denotes the shift operator in $k$ (and similarly $\hat{S}$ and $\dot{S}$ represent shifts in $l$ resp. $m$ ), and 1 is the identity. This gives $(1-\tilde{S}) \ln (p)=(1-\hat{S}) \ln (q)=(1-\dot{S}) \ln (v)$, which can be written in quotient form,

$$
\frac{\tilde{p}}{p}=\frac{\hat{q}}{q}=\frac{\dot{v}}{v}
$$

As (7) contains only two independent equations the system of equations for $p, q, v$ defined by (6) and (7) can be referred to as a QQD-system.

Similarly, we can write the dual AKP equation in variables

$$
u=\frac{\tilde{\tau} \dot{\tau}}{\tau \dot{\tau}}, \quad z=\frac{\hat{\tau} \dot{\tau}}{\tau \dot{\tau}}, \quad w=\frac{\dot{\tau} \hat{\tilde{\tau}}}{\tilde{\tau} \hat{\tau}}
$$

and the variable $v$ introduced above. We have $u=-P_{31}=Q_{31}, z=P_{32}=-Q_{32}$, and $w=R_{33}$. The third conservation law becomes

$$
a_{1}(\hat{u}-\tilde{u})+a_{2}(\tilde{z}-\hat{z})+a_{3}(w-\dot{w})+a_{4}(v-\dot{v})=0 .
$$

Taking logarithms we find

$$
\ln (u)=\frac{(\tilde{S}-1)(\tilde{S}-\dot{S})}{\tilde{S}} c, \quad \ln (z)=\frac{(\hat{S}-1)(\hat{S}-\dot{S})}{\hat{S}} c, \quad \ln (w)=\frac{(\tilde{S}-\dot{S})(\hat{S}-\tilde{S})}{\dot{S}} c .
$$

One can now derive quotient equations which are either ratios of quadratic terms

$$
\frac{\hat{\tilde{\tilde{u}}} \hat{\tilde{u}}}{\dot{\hat{\tilde{u}}} \hat{\tilde{u}}}=\frac{\hat{\tilde{\tilde{z}}} \dot{\tilde{z}}}{\dot{\dot{\tilde{z}}} \hat{\tilde{z}}}=\frac{\dot{\tilde{\tilde{w}}} \dot{\tilde{w}}}{\dot{\tilde{w}} \dot{\hat{w}}}=\frac{\hat{\tilde{v}} \dot{\dot{v}}}{\dot{\dot{v}} \dot{\hat{v}}},
$$

or ratios of linear terms

$$
\frac{\tilde{\hat{u}}}{\tilde{u}}=\frac{\tilde{v}}{\dot{v}}, \quad \frac{\tilde{\hat{u}}}{\dot{\tilde{u}}}=\frac{\dot{\tilde{w}}}{\dot{w}}, \quad \frac{\tilde{\hat{z}}}{\hat{z}}=\frac{\hat{v}}{\dot{v}}, \quad \frac{\tilde{\hat{z}}}{\dot{\hat{z}}}=\frac{\dot{\hat{w}}}{\dot{w}},
$$

of which only three are independent. In the sequel, we will refer to the system of quotient and difference equations $(9)$ and $(10)$ as the $\mathrm{Q}^{3} \mathrm{D}$-system.

\section{The $N$-soliton solution}

\section{1-soliton}

Equation (1) admits the 1-soliton solution $\tau_{k, l, m}=1+c_{1} x_{1}^{k} y_{1}^{l} z_{1}^{m}$ with dispersion relation $Q_{1}=0$, where

$$
\begin{aligned}
Q_{i}= & y_{i} z_{i}\left(x_{i}-1\right)\left(x_{i}-y_{i}\right)\left(x_{i}-z_{i}\right) a_{1}+x_{i} z_{i}\left(y_{i}-1\right)\left(y_{i}-x_{i}\right)\left(y_{i}-z_{i}\right) a_{2} \\
& +x_{i} y_{i}\left(z_{i}-1\right)\left(z_{i}-x_{i}\right)\left(z_{i}-y_{i}\right) a_{3}+x_{i} y_{i} z_{i}\left(x_{i}-1\right)\left(y_{i}-1\right)\left(z_{i}-1\right) a_{4} .
\end{aligned}
$$

In the sequel we will use the following notation, $x_{i j}=x_{i} x_{j}, c_{i j}=c_{i} c_{j}$, and if $Q_{i}=Q\left(x_{i}, y_{i}, z_{i}\right)$ then $Q_{i j}=$ $Q\left(x_{i j}, y_{i j}, z_{i j}\right)$.

\section{2-soliton}

Equation (1) admits the 2-soliton solution

$$
\tau_{k, l, m}=1+c_{1} x_{1}^{k} y_{1}^{l} z_{1}^{m}+c_{2} x_{2}^{k} y_{2}^{l} z_{2}^{m}+c_{1} c_{2} R_{12} x_{12}^{k} y_{12}^{l} z_{12}^{m}
$$

where $Q_{1}=Q_{2}=0$,

$$
R_{i j}=\frac{a_{1} S_{1}^{i j}+a_{2} S_{2}^{i j}+a_{3} S_{3}^{i j}+a_{4} S_{4}^{i j}}{Q_{i j}},
$$


with

$$
\begin{aligned}
S_{1}^{i j}= & \left(\left(x_{i}-x_{j}\right)\left(x_{j} y_{i}-y_{j} x_{i}\right)\left(x_{i j}-z_{i j}\right)+\left(x_{i}-x_{j}\right)\left(x_{j} z_{i}-z_{j} x_{i}\right)\left(x_{i j}-y_{i j}\right)\right. \\
& \left.+\left(x_{j} z_{i}-z_{j} x_{i}\right)\left(x_{j} y_{i}-y_{j} x_{i}\right)\left(1-x_{i j}\right)\right) y_{i j} z_{i j} \\
S_{4}^{i j}= & \left(\left(1-x_{i j}\right)\left(y_{i}-y_{j}\right)\left(z_{i}-z_{j}\right)+\left(x_{i}-x_{j}\right)\left(1-y_{i j}\right)\left(z_{i}-z_{j}\right)\right. \\
& \left.+\left(x_{i}-x_{j}\right)\left(y_{i}-y_{j}\right)\left(1-z_{i j}\right)\right) x_{i j} y_{i j} z_{i j}
\end{aligned}
$$

and $S_{k}^{i j}$ for $k=2$, resp. $k=3$, are obtained from $S_{1}^{i j}$ by interchanging the symbols $x$ and $y$, respectively $x$ and $z$. This has been checked by direct computation, using a Groebner basis in Maple [25].

\section{$N$-soliton}

Let $P(N)$ denote the powerset of the string $12 \ldots N$, e.g. we write

$$
P(3)=\{\varepsilon, 1,2,3,12,23,13,123\}
$$

where $\varepsilon$ is the empty string, and let $P_{2}(S)$ be the subset of the powerset of a string $S$ containing all 2-letter substrings, e.g.

$$
P_{2}(123)=\{12,23,13\} .
$$

Conjecture 1 Equation (1) admits the following $N$-soliton solution:

$$
\tau_{k, l, m}=\sum_{w \in P(N)}\left(\prod_{v \in P_{2}(w)} R_{v}\right) c_{w} x_{w}^{k} y_{w}^{l} z_{w}^{m}, \quad \text { with } Q_{i}=0, i \in\{1,2, \ldots, N\} .
$$

Note that in the above formula $c_{\varepsilon}=x_{\varepsilon}=\cdots=1$ is understood. The formula can be computationally checked as follows: Taking particular values for $a_{1}, a_{2}, a_{3}$ and $a_{4}$, one can find rational points $p_{i}=\left(x_{i}, y_{i}, z_{i}\right) \in \mathbb{Q}^{3}$ such that $Q_{i}=0$. Using $N \in \mathbb{N}$ points, one substitutes the $N$-soliton solution, which contains $N$ arbitrary constants $c_{1}, \ldots, c_{N}$, into the equation for fixed points $(k, l, m) \in \mathbb{Z}^{3}$. For example, taking $\left(a_{1}, a_{2}, a_{3}, a_{4}\right)=(1,2,3,2)$ the following points

$$
\begin{array}{lll}
p_{1}=(2,4,2 / 3), & p_{2}=(6,21,-14), & p_{3}=(7,14,-6), \\
p_{4}=(8,15,-40 / 9), & p_{5}=(14,80,-560), & p_{6}=(18,120,-15 / 2)
\end{array}
$$

satisfy $Q_{i}=0$. Taking $k=-2, l=1, m=3$ one needs to verify that

$$
\begin{aligned}
& \tau_{-3,2,4} \tau_{-1,1,3} \tau_{-1,1,4} \tau_{-1,2,3}+2 \tau_{-2,1,3} \tau_{-2,2,4} \tau_{-1,1,4} \tau_{-1,2,3}-\tau_{-2,1,4} \tau_{-2,2,3} \tau_{-2,2,4} \tau_{0,1,3} \\
& -2 \tau_{-2,1,4} \tau_{-2,2,3} \tau_{-1,1,3} \tau_{-1,2,4}+3 \tau_{-2,1,4} \tau_{-2,2,4} \tau_{-1,1,4} \tau_{-1,2,2}-2 \tau_{-2,1,4} \tau_{-2,3,3} \tau_{-1,1,3} \tau_{-1,1,4} \\
& -3 \tau_{-2,1,5} \tau_{-2,2,3} \tau_{-1,1,3} \tau_{-1,2,3}+2 \tau_{-2,2,3} \tau_{-2,2,4} \tau_{-1,0,4} \tau_{-1,2,3}
\end{aligned}
$$

vanishes. Using the above 6 points $p_{i}$ the value of the 6 -soliton solution at $(k, l, m)=(-3,2,4)$ is

$$
\begin{aligned}
\tau_{-3,2,4} & =1+\frac{32 c_{1}}{81}+\frac{235298 c_{2}}{3}+\frac{5184 c_{3}}{7}+\frac{125000 c_{4}}{729}-\frac{9973408256.10^{8} c_{1} c_{2} c_{3} c_{4}}{2850829229061}+\frac{17537436614656.10^{14} c_{1} c_{4} c_{5}}{304882184692881} \\
& -\frac{286643773308928.10^{13} c_{2} c_{4} c_{5}}{5379614362287}-\frac{31023435087872.10^{14} c_{3} c_{4} c_{5}}{1827893357279451}-\frac{8355684882055168.10^{7} c_{1} c_{2} c_{5}}{3557331}-\frac{244.10^{6} c_{1} c_{4}}{2735937} \\
& +\frac{419082155327488.10^{10} c_{1} c_{3} c_{5}}{79592065203}-\frac{25192657019901837312.10^{7} c_{2} c_{3} c_{5}}{414577637413}+\frac{15625 c_{6}}{2}+229376.10^{6} c_{5}+\frac{430515.10^{5} c_{3} c_{6}}{313747} \\
& +\frac{1220703125 c_{4} c_{6}}{34992}-\frac{6272.10^{13} c_{5} c_{6}}{339}+\frac{5.10^{5} c_{1} c_{6}}{1539}-\frac{1838265625 c_{2} c_{6}}{422}+\frac{4563788408614224681500672.10^{17} c_{1} c_{2} c_{3} c_{4} c_{5}}{3335745327609453768757318923}
\end{aligned}
$$




$$
\begin{aligned}
& -\frac{1075648.10^{6} c_{2} c_{3} c_{4}}{253204479}+\frac{9415684768.10^{6} c_{1} c_{2} c_{4}}{1595051271}-\frac{74176.10^{8} c_{1} c_{3} c_{4}}{10556764911}+\frac{359696691200 c_{1} c_{2} c_{3}}{13161}-\frac{323830284288.10^{6} c_{2} c_{5}}{367} \\
& +\frac{68956750243187158016.10^{25} c_{1} c_{2} c_{3} c_{4} c_{5} c_{6}}{13520106051588549460696520569492377}+\frac{203190312540790063104.10^{17} c_{1} c_{2} c_{3} c_{5} c_{6}}{22721904043520272815643}-\frac{270945647.10^{21} c_{2} c_{4} c_{5} c_{6}}{3405295891327671} \\
& +\frac{2558749998443072.10^{22} c_{1} c_{2} c_{4} c_{5} c_{6}}{87172894850121902722923}+\frac{512857367787136.10^{25} c_{1} c_{3} c_{4} c_{5} c_{6}}{17456412275319361592913150717}-\frac{5065859375.10^{8} c_{1} c_{3} c_{4} c_{6}}{1419494280227793}-\frac{8.10^{6} c_{3} c_{4}}{22869} \\
& +\frac{1197498441289024.10^{22} c_{2} c_{3} c_{4} c_{5} c_{6}}{3344920733568156174088032717}+\frac{486525134375.10^{10} c_{1} c_{2} c_{3} c_{4} c_{6}}{3851564365825970013}+\frac{433061888.10^{7} c_{1} c_{5}}{29079}-\frac{5190429687500 c_{3} c_{4} c_{6}}{3075034347} \\
& -\frac{3785197879296.10^{7} c_{3} c_{5}}{1802479}-\frac{152276992.10^{13} c_{4} c_{5}}{226286703}-\frac{23683072.10^{14} c_{1} c_{5} c_{6}}{187297839}+\frac{1353499793462147416064.10^{14} c_{1} c_{2} c_{4} c_{5}}{7248099679897056849} \\
& -\frac{24118045.10^{5} c_{1} c_{2} c_{6}}{324729}+\frac{34110994995.10^{5} c_{2} c_{3} c_{6}}{5926981771}-\frac{297851562500 c_{1} c_{4} c_{6}}{155948409}+\frac{287875.10^{18} c_{4} c_{5} c_{6}}{2036580327}+\frac{308710976 c_{1} c_{2}}{243} \\
& +\frac{143614501953125 c_{2} c_{4} c_{6}}{358705908}-\frac{8504.10^{8} c_{1} c_{3} c_{6}}{38590881}-\frac{4427367168.10^{13} c_{2} c_{5} c_{6}}{8750381}-\frac{972800 c_{1} c_{3}}{861}-\frac{224599144.10^{8} c_{1} c_{2} c_{3} c_{6}}{5926981771} \\
& -\frac{1916804736 c_{2} c_{3}}{4387}-\frac{117649.10^{6} c_{2} c_{4}}{425007}+\frac{929743819565759987712.10^{10} c_{1} c_{2} c_{3} c_{5}}{148833371831267}+\frac{5239147585536.10^{14} c_{3} c_{5} c_{6}}{1304163853181} \\
& -\frac{16971308117950201856.10^{17} c_{1} c_{3} c_{4} c_{5}}{302920719026139857929671}+\frac{8342573976349835264.10^{14} c_{2} c_{3} c_{4} c_{5}}{825274865866379324583}-\frac{183176122990592.10^{17} c_{1} c_{3} c_{5} c_{6}}{172763889794740251} \\
& -\frac{66307976.10^{22} c_{1} c_{4} c_{5} c_{6}}{52134853582482651}+\frac{4984888671875.10^{4} c_{2} c_{3} c_{4} c_{6}}{342087606876807}-\frac{52304285961241509888.10^{14} c_{2} c_{3} c_{5} c_{6}}{63292211820390732077} \\
& +\frac{8906247704.10^{22} c_{3} c_{4} c_{5} c_{6}}{105336010499942922777}-\frac{574687791015625.10^{4} c_{1} c_{2} c_{4} c_{6}}{6394560545439}-\frac{228475758493696.10^{14} c_{1} c_{2} c_{5} c_{6}}{1611531417627},
\end{aligned}
$$

and we obtain similar expressions for the values of the 6 -soliton solution at the other 13 lattice points of the stencil, cf. Figure 1. Substituting these expressions into (12) gives 0 . This has been checked also for values of $a_{i}$, other point $p_{j}$ and other values for $k, l, m$.

We have also performed another computational verification, this time of the 3-soliton solution. Starting with expressions for $p_{1}, p_{2}, p_{3}$ of the form $p_{i}=b_{i} x+c_{i}$ where $b_{i}, c_{i} \in \mathbb{Q}$ are randomly chosen and $x$ is a parameter, we have solved the linear system $Q_{12}=Q_{13}=Q_{23}=0$ for $a_{1}, a_{2}, a_{3}, a_{4}$, and verified the solution for a range of values for $k, l, m$.

In Figure 2 we have plotted two cross sections of a three soliton solution.
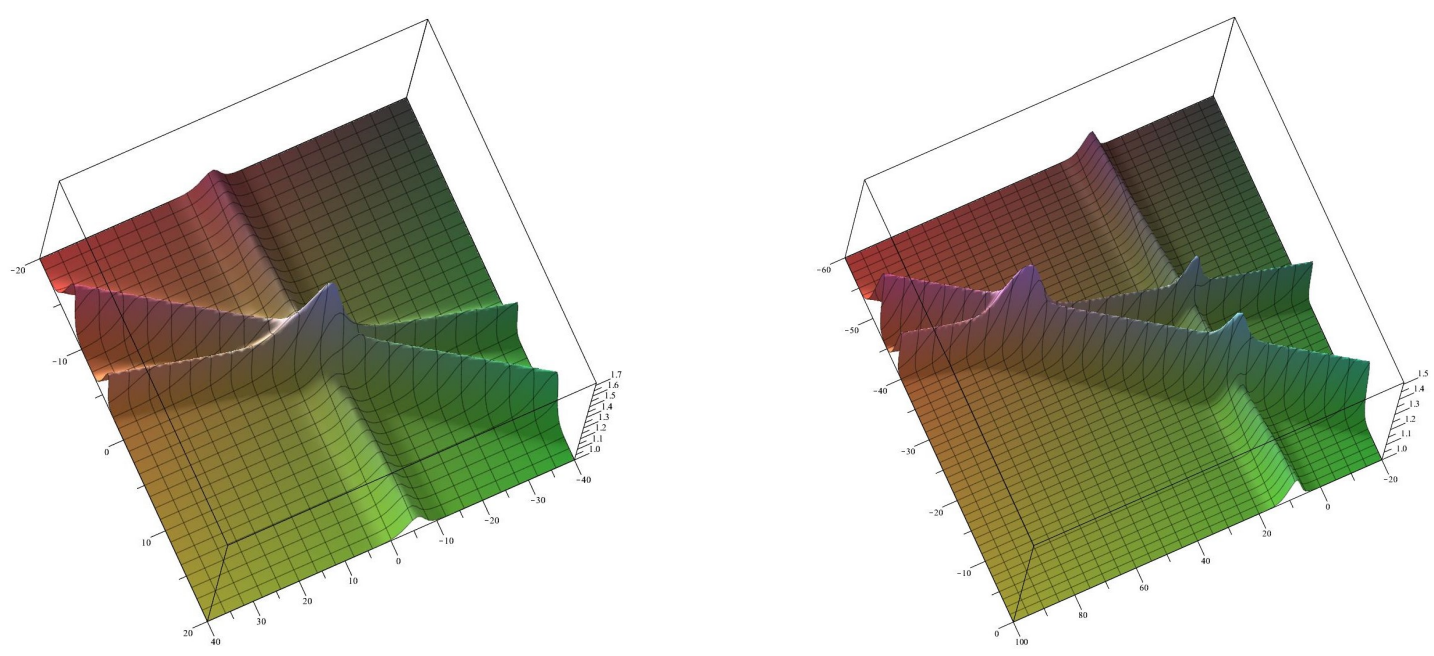

Figure 2: Two cross sections, $m=0$ resp. $m=50$, of the function $u$ defined in (8) where $\tau$ is the three soliton solution of dual AKP with $\left(a_{1}, a_{2}, a_{3}, a_{4}\right)=(1,2,3,2)$ and $p_{1}=\left(\frac{1}{5}, \frac{12}{13}, \frac{18}{65}\right), p_{2}=\left(\frac{11}{31}, \frac{15}{32}, \frac{495}{496}\right), p_{3}=\left(2,4, \frac{2}{3}\right)$, and $c_{1}=c_{2}=c_{3}=1$. 


\section{$5 \quad$ Laurent property}

Consider an ordinary difference equation of order $d$,

$$
\tau_{n}=\frac{P\left(\tau_{n-d}, \ldots, \tau_{n-1}\right)}{Q\left(\tau_{n-d}, \ldots, \tau_{n-1}\right)}
$$

where $P$ is a polynomial and $Q$ is a monomial. Let $\mathcal{R}$ be the ring of coefficients. From a set of $d$ initial values $U=\left\{\tau_{k}\right\}_{0 \leq k<d}$, one finds $\tau_{n}$ as rational functions of the initial values, given by

$$
\tau_{n}=\frac{p_{n}\left(\tau_{0}, \ldots, \tau_{d-1}\right)}{q_{n}\left(\tau_{0}, \ldots, \tau_{d-1}\right)},
$$

with greatest common divisor $\operatorname{gcd}\left(p_{n}, q_{n}\right)=1$. By definition, if $q_{n} \in \mathcal{R}[U]$ is a monomial for all $n \geq 0$, then (13) has the Laurent property. The first examples of recurrences with the Laurent property were discovered by Michael Somos in the 1980s [12]. Since then many more have been found [2, 6, 8, 15, 24], and the Laurent property is a central feature of cluster algebras [9, 10. In [27, Definition 2.11] the author defines the Laurent property for discrete bilinear equations. The idea is that a lattice equation has the Laurent property if all good initial value problems have the Laurent property. The author points out that not all well-posed, cf. [34, initial value problems are good. Certainly, the initial value problems obtained from (doubly periodic) reductions given below, see (15), are good.

In [14 a more specific Laurent property was introduced, where the terms are Laurent polynomials in some of the variables but polynomial in others. The form of 13 guarantees that all components $q_{n}$ are monomials for $0 \leq n \leq d$. Suppose these monomials depend on a subset of the initial values $V \subset U$, specified by a set of superscripts $I \subset\{1, \ldots, d\}$. The following conditions guarantee that $q_{n}$ is a monomial $\in \mathcal{R}[V]$ for all $i$ and $n \geq 0$, cf. [14, Theorem 2].

Theorem 2 Suppose that $q_{d}$ is a monomial in $\mathcal{R}[V]$. If $p_{d}$ is coprime to $p_{d+k}$ for all $k=1, \ldots, d$, and $q_{m} \in \mathcal{R}[V]$ is a monomial for $d+1 \leq m \leq 2 d$, then (13) has the following Laurent property: all iterates are Laurent polynomials in the variables from $V$ and they are polynomial in the remaining variables from $W=U \backslash V$.

Introducing the variable $n=z_{1} k+z_{2} l+z_{3} m$, where we take $z_{1}, z_{2}, z_{3}$ to be non-negative integers such that $\operatorname{gcd}\left(z_{1}, z_{2}, z_{3}\right)=1$, and performing a reduction $\tau_{k, l, m} \rightarrow \tau_{n}$, one obtains the ordinary difference equation

$$
\begin{aligned}
0= & a_{1}\left(\tau_{n+z_{1}} \tau_{n+z_{1}+z_{2}} \tau_{n+z_{1}+z_{3}} \tau_{n-z_{1}+z_{2}+z_{3}}-\tau_{n+2 z_{1}} \tau_{n+z_{2}} \tau_{n+z_{3}} \tau_{n+z_{2}+z_{3}}\right) \\
& +a_{2}\left(\tau_{n+z_{2}} \tau_{n+z_{1}+z_{2}} \tau_{n+z_{2}+z_{3}} \tau_{n+z_{1}-z_{2}+z_{3}}-\tau_{n+z_{1}} \tau_{n+2} z_{2} \tau_{n+z_{3}} \tau_{n+z_{1}+z_{3}}\right) \\
& +a_{3}\left(\tau_{n+z_{3}} \tau_{n+z_{1}+z_{3}} \tau_{n+z_{2}+z_{3}} \tau_{n+z_{1}+z_{2}-z_{3}}-\tau_{n+z_{1}} \tau_{n+z_{2}} \tau_{n+2} z_{3} \tau_{n+z_{1}+z_{2}}\right) \\
& +a_{4}\left(\tau_{n} \tau_{n+z_{1}+z_{2}} \tau_{n+z_{1}+z_{3}} \tau_{n+z_{2}+z_{3}}-\tau_{n+z_{1}} \tau_{n+z_{2}} \tau_{n+z_{3}} \tau_{n+z_{1}+z_{2}+z_{3}}\right)
\end{aligned}
$$

which has order

$$
d=\max \left(2 z_{1}, 2 z_{2}, 2 z_{2}, z_{1}+z_{2}+z_{3}\right)-\min \left(0, z_{1}+z_{2}-z_{3}, z_{1}+z_{3}-z_{2}, z_{2}+z_{3}-z_{1}\right) .
$$

Conjecture 3 The iterates $\tau_{n}$ are Laurent polynomials in the initial values $\tau_{i}$, with $i=p, p+1, \ldots, d-p-1$ where

$$
p=\min \left(z_{1}, z_{2}, z_{2}\right)-\min \left(0, z_{1}+z_{2}-z_{3}, z_{1}+z_{3}-z_{2}, z_{2}+z_{3}-z_{1}\right)
$$

and polynomial in the others, $\tau_{0}, \tau_{1}, \ldots, \tau_{p-1}, \tau_{d-p}, \ldots, \tau_{d-2}, \tau_{d-1}$.

This conjecture has been proven, using Theorem 2 and [25], for $z_{1}=z_{2}=1,1 \leq z_{3} \leq 20$, for $z_{1}=1, z_{2}=2, z_{3}=$ 3 , and some but not all of the conditions of Theorem 2 have been verified for all co-prime $z_{1}<z_{2}<z_{3} \leq 10$.

\section{Degree growth}

Given an ordinary difference equation of the form 13 one can define an integer sequence $\left\{d_{n}^{p}\right\}_{n=0}^{\infty}$ where $d_{n}^{p}$ denotes the degree of the polynomial $p_{n}$ defined by (14). According to the degree growth conjecture [7, 17] we have

- growth is linear in $n \Longrightarrow$ equation is linearizable.

- growth is polynomial in $n \Longrightarrow$ equation is integrable. 
- growth is exponential in $n \Longrightarrow$ equation is non-integrable.

Conjecture 4 For all positive integers $z_{1}, z_{2}, z_{3}$ such that $\operatorname{gcd}\left(z_{1}, z_{2}, z_{3}\right)=1$ equation (15) has quadratic growth.

We have verified the following. Choosing (randomly) rational values for the coefficients $a_{i}$, starting with rational initial values $\tau_{0}, \ldots, \tau_{d-2}$ and letting $\tau_{d-1}=a+b x$, where $a, b$ are rational values and $x$ a parameter, we have calculated up to a 150 iterates until the degree (in $x$ ) exceeded 250. Taking the second difference of the degree sequence yielded a periodic sequence in almost all cases with $1 \leq z_{1} \leq 4,1 \leq z_{2} \leq z_{3} \leq 7$. In two cases more iterations were required. Keeping the maximal degree fixed at 500, for $z=(1,1,7)$ we calculated 370 iterations and found that the period of the second difference is 259 , for $z=(3,7,7)$ we calculated 354 iterations and found that the period of the second difference is 240 . Curiously, the leading order terms are all of the form $\left(M_{z_{2}, z_{3}}^{z_{1}}\right)^{-1} n^{2}$ with

$$
\begin{aligned}
M^{1}=\left[\begin{array}{ccccccc}
2 & 4 & 15 & 40 & 85 & 156 & 259 \\
4 & 7 & 12 & 25 & 60 & 94 & 172 \\
15 & 12 & 16 & 24 & 40 & 76 & 150 \\
40 & 25 & 24 & 29 & 40 & 60 & 108 \\
85 & 60 & 40 & 40 & 46 & 60 & 82 \\
156 & 94 & 76 & 60 & 60 & 67 & 84 \\
259 & 172 & 150 & 108 & 82 & 84 & 92
\end{array}\right], \quad M^{2}=\left[\begin{array}{ccccccc}
4 & 7 & 12 & 25 & 60 & 94 & 172 \\
7 & x & 15 & x & 40 & x & 154 \\
12 & 15 & 28 & 25 & 60 & 55 & 132 \\
25 & x & 25 & x & 40 & x & 76 \\
60 & 40 & 60 & 40 & 84 & 60 & 140 \\
94 & x & 55 & x & 60 & x & 82 \\
172 & 154 & 132 & 76 & 140 & 82 & 172
\end{array}\right], \\
M^{3}=\left[\begin{array}{ccccccc}
15 & 12 & 16 & 24 & 40 & 76 & 150 \\
12 & 15 & 28 & 25 & 60 & 55 & 132 \\
16 & 28 & x & 40 & 40 & x & 77 \\
24 & 25 & 40 & 69 & 60 & 55 & 168 \\
40 & 60 & 40 & 60 & 114 & 76 & 76 \\
76 & 55 & x & 55 & 76 & x & 108 \\
150 & 132 & 77 & 168 & 76 & 108 & 240
\end{array}\right], \quad M^{4}=\left[\begin{array}{ccccccc}
25 & x & 25 & x & 40 & x & 76 \\
24 & 25 & 40 & 69 & 60 & 55 & 168 \\
29 & x & 69 & x & 85 & x & 77 \\
40 & 40 & 60 & 85 & 136 & 94 & 132 \\
60 & x & 55 & x & 94 & x & 150 \\
108 & 76 & 168 & 77 & 132 & 150 & 296
\end{array}\right],
\end{aligned}
$$

where $x$ indicates that $\operatorname{gcd}\left(z_{1}, z_{2}, z_{3}\right)>1$.

\section{Reductions to $2 \mathrm{D}$ integrable lattice equations}

We give some reductions to integrable $2 \mathrm{D}$ lattice equations known in the literature.

- Setting ${ }^{\cdot}{ }^{\wedge}, u=e, v=\tilde{q}, z=w=0$ and $a_{1}+a_{4}=0$ the $\mathrm{Q}^{3} \mathrm{D}$-system reduces to Rutishauser's QD-algorithm

$$
\tilde{e}+\tilde{q}=\hat{e}+\tilde{\hat{q}}, \quad \hat{e} \hat{q}=e \tilde{q} .
$$

- Taking $z=a_{2}=0$ and $a_{1}=a_{3}=-a_{4}=1$ and introducing variables $i=k-l, j=3 l+m, \tau_{k, l, m}=\Delta_{i}^{j}$, equation (1) reduces to the higher analogue of the discrete-time Toda (HADT) equation [32, Equation (3.18)],

$$
\begin{aligned}
& \Delta_{i+1}^{j}\left(\Delta_{i-2}^{j+4} \Delta_{i+1}^{j+1} \Delta_{i}^{j+3}-\Delta_{i}^{j+2} \Delta_{i-1}^{j+3} \Delta_{i}^{j+3}+\Delta_{i-1}^{j+3} \Delta_{i}^{j+1} \Delta_{i}^{j+4}\right) \\
& =\Delta_{i-1}^{j+4}\left(-\Delta_{i+2}^{j} \Delta_{i}^{j+1} \Delta_{i-1}^{j+3}+\Delta_{i}^{j+2} \Delta_{i}^{j+1} \Delta_{i+1}^{j+1}-\Delta_{i}^{j} \Delta_{i+1}^{j+1} \Delta_{i}^{j+3}\right),
\end{aligned}
$$

and the $\mathrm{Q}^{3} \mathrm{D}$-system reduces to the QQD-system [32, Equation (1.4)],

$$
\begin{aligned}
u_{i, 3+j}+v_{i+1, j+1}+w_{i+1, j} & =u_{i+2, j}+v_{i+1, j}+w_{i+1, j+1} \\
u_{i, 3+j} v_{i, j+1} & =u_{i+1, j} v_{i+1, j} \\
u_{i, 3+j} w_{i, j+1} & =u_{i+1, j+1} w_{i+1, j+1} .
\end{aligned}
$$


- By introducing some special bi-orthogonal polynomials, in 3 the so-called discrete hungry quotient-difference (dhQD) algorithm and a system related to the QD-type discrete hungry Lotka-Volterra (QD-type dhLV) system have been derived, as well as hungry forms of the HADT-equation (hHADT) and the QQD scheme (hQQD). These systems are all reductions of the $\mathrm{Q}^{3} \mathrm{D}$ system, or of the dual to the AKP equation, (1).

Setting $z=w=0, \tilde{u}=q, v=e$ and introducing $i=k, j=p l+m$ we get QD-type $\operatorname{dhLV}$ [3. Equations $(6,7)]$,

$$
\begin{aligned}
e_{i, j}+q_{i, j} & =e_{i, j+1}+q_{i-1, j+p} \\
e_{i, j+1} q_{i, j+p} & =e_{i+1, j} q_{i, j} .
\end{aligned}
$$

Setting $z=w=0, \tilde{u}=q, v=e$ and introducing $i=k, j=l+p m$ we get dhQD [3, Equations $(9,10)]$,

$$
\begin{aligned}
e_{i, j}+q_{i, j} & =e_{i, j+p}+q_{i-1, j+1} \\
e_{i, j+p} q_{i, j+1} & =e_{i+1, j} q_{i, j} .
\end{aligned}
$$

With $z=0$ the reduction $i=k-l, j=(p+2) l+p m$ yield hQQD [3], Equation (23)],

$$
\begin{aligned}
u_{i, j+p+2}+v_{i+1, j+p}+w_{i+1, j} & =u_{i+2, j}+v_{i+1, j}+w_{i+1, j+p} \\
u_{i, j+p+2} v_{i, j+p} & =u_{i+1, j} v_{i+1, j} \\
u_{i, j+2} w_{i, j} & =u_{i+1, j} w_{i+1, j} .
\end{aligned}
$$

Performing the same reduction on equation (1), with $a_{2}=0$, gives the hHADT equation [3, Equation (18)],

$$
\begin{aligned}
& \left(\Delta_{i-2}^{j+2 p+2} \Delta_{i+1}^{j+p} \Delta_{i}^{j+p+2}-\Delta_{i}^{j+2 p} \Delta_{i-1}^{j+p+2} \Delta_{i}^{j+p+2}+\Delta_{i}^{j+2 p+2} \Delta_{i}^{j+p} \Delta_{i-1}^{j+p+2}\right) \Delta_{i+1}^{j} \\
& =\left(\Delta_{i+2}^{j} \Delta_{i}^{j+p} \Delta_{i-1}^{j+p+2}-\Delta_{i}^{j+2} \Delta_{i}^{j+p} \Delta_{i+1}^{j+p}+\Delta_{i}^{j} \Delta_{i+1}^{j+p} \Delta_{i}^{j+p+2}\right) \Delta_{i-1}^{j+2 p+2} .
\end{aligned}
$$

\section{Conclusion}

In this paper we have generalized the concept of duality introduced in 29] for ordinary difference equations (O $\Delta \mathrm{Es})$ to the realm of lattice equations (P $\Delta \mathrm{Es})$. The dAKP equation (1) and the AKP equation (2) are dual to each other. Generally speaking, dual equations to integrable equations do not need to be integrable themselves; the only thing that is guaranteed is the existence of integrals (for $\mathrm{O} \Delta \mathrm{Es}$ ), or conservation laws (for $\mathrm{P} \Delta \mathrm{Es}$ ). However, our equation (2) unifies a number of known (hierarchies of) integrable 2D lattice equations, which arise as reductions. Together with the support we have provided for our conjectures, that equation (1) admits an $N$-soliton solution, and its reductions have the Laurent property and zero algebraic entropy, we believe it is a new integrable 3D lattice equation.

\section{Acknowledgements}

This research was supported by the Australian Research Council [DP140100383], by the NSF of China [No. 11371241, 11631007], and by two La Trobe University China Strategy Implementation Grants.

\section{References}

[1] V.E Adler, A.I. Bobenko, Yu.B. Suris, Classification of integrable discrete equations of octahedron type, Int Math Res Not 2012 (2012) 1822-1889.

[2] J. Alman, C. Cuenca, J. Huang, Laurent Phenomenon Sequences, J Algebr Comb 43 (2016) 589-633.

[3] X.K. Chang, X.M. Chen, X.B. Hu, H.W. Tam, About several classes of bi-orthogonal polynomials and discrete integrable systems, J Phys A: Math Theo 48 (2015) 015204 (17pp).

[4] D.K. Demskoi, D.T. Tran, P.H. van der Kamp, G.R.W. Quispel, A novel $n$th order difference equation that may be integrable, J Phys A: Math Theor 45 (2012) 135202 (10pp).

[5] D.K. Demskoi, P.H. van der Kamp, G.R.W. Quispel, Are dual difference equations integrable? (2015) unpublished work. 
[6] S.B. Ekhad, D. Zeilberger, How to generate as many Somos-like miracles as you wish, J Differ Equ Appl 20 (2014) 852-858.

[7] G. Falqui, C-M. Viallet, Singularity, complexity, and quasi-integrability of rational mappings, Comm Math Phys 154 (1993) 111-125.

[8] S. Fomin, A. Zelevinsky, The Laurent Phenomenon, Adv Appl Math 28 (2002) 119-144.

[9] S. Fomin, A. Zelevinsky, Cluster algebras IV: coefficients, Comp Math 143 (2007) 112-164.

[10] S. Fomin, M. Shapiro, D. Thurston, Cluster algebras and triangulated surfaces. Part I: Cluster complexes, Acta Mathematica 201 (2008) 83-146.

[11] W. Fu and F.W. Nijhoff, Direct linearizing transform for three-dimensional discrete integrable systems: the lattice AKP, BKP and CKP equations, Proc R Soc A 473 (2017) 20160915 (22pp).

[12] D. Gale, The strange and surprising saga of the Somos sequences, Math Intell 13 (1991) 40-42.

[13] B. Grammaticos, A. Ramani, V. Papageorgiou, J. Satsuma and R. Willox, Constructing lump-like solutions of the Hirota-Miwa equation, J Phys A: Math Theor 40 (2007) 12619-12627.

[14] K. Hamad, A.N.W. Hone, P.H. van der Kamp, G.R.W. Quispel, QRT maps and related Laurent systems, Adv App Math 96 (2018) 216-248.

[15] K. Hamad, P.H. van der Kamp, From discrete integrable equations to Laurent recurrences, J Differ Equ Appl 22 (2016) 789-816.

[16] J. Hietarinta, N. Joshi, F.W. Nijhoff, Discrete Systems and Integrability, Camb Univ Press, 2016.

[17] J. Hietarinta, C-M. Viallet, Searching for integrable lattice maps using factorization. J Phys A: Math Theor 40 (2007) 12629-12643.

[18] R. Hirota, Discrete analogue of a generalized Toda equation, J Phys Soc Japan 50 (1981) 3785-3791.

[19] R. Hirota, Nonlinear partial difference equations, I-III, J Phys Soc Japan 43 (1977) 1424-1433, 2074-2089.

[20] A.N.W. Hone, T.E. Kouloukas, G.R.W. Quispel, Some integrable maps and their Hirota bilinear forms, J Phys A: Math Theor 51 (2017) 044004 (30pp).

[21] N. Joshi, C.-M. Viallet, Rational maps with invariant surfaces, arXiv:1706.00173 [nonl.SI].

[22] R.M. Kashaev, On discrete three-dimensional equations associated with the local Yang-Baxter relation, Lett Math Phys 38 (1996) 389-397.

[23] A.D. King, W.K. Schief, Bianchi Hypercubes and a Geometric Unification of the Hirota and Miwa Equations, Int Math Res Not 2015 (2015) 6842-6878.

[24] T. Lam, P. Pylyavskyy, Laurent phenomenon algebras, Cambridge J Math 4 (2016) 121-162.

[25] Maple 2016, http://www.maplesoft.com/products/Maple/index.aspx

[26] K. Maruno, G.R.W.Quispel, Construction of integrals of higher-order mappings, J Phys Soc Japan 75 (2006) 123001 (5pp).

[27] T. Mase, The Laurent Phenomenon and Discrete Integrable Systems, RIMS Kôkyûroku Bessatsu B41 (2013) $43-64$.

[28] T. Miwa, On Hirota's difference equations, Proc Japan Acad 58A (1982) 9-12.

[29] G.R.W. Quispel, H.W. Capel, J.A.G. Roberts, Duality for discrete integrable systems, J Phys A: Math Gen 38 (2005) 3965-3980.

[30] H. Rutishauser, Der Quotienten-Differenzen Algorithmus, Z Angew Math Phys 5 (1954) 233-251.

[31] W.K. Schief, Lattice geometry of the discrete Darboux, KP, BKP and CKP equations. Menelaus' and Carnot's theorem, J Nonl Math Phys 10 (2003) 194-208. 
[32] P.E. Spicer, F.W. Nijhoff, P.H. van der Kamp, Higher analogues of the discrete-time Toda equation and the quotient-difference algorithm, Nonlinearity 24 (2011) 2229-2263.

[33] S.P. Tsarev, T. Wolf, Hyperdeterminants as integrable discrete systems, J Phys A: Math Theor 42 (2009) 454023 (9pp).

[34] P.H. van der Kamp, Initial value problems for quad equations, J Phys A: Math Theor 48 (2015) 065204 (20pp). 